\title{
Urinary Tract Infection in Women Over the Age of 65: Is Age Alone a Marker of Complication?
}

\author{
Michael L. Grover, DO, Jesse D. Bracamonte, DO, Anup K. Kanodia, MD, \\ Frederick D. Edwards, MD, and Amy L. Weaver, MS
}

Background: We were interested to know if our older female patients with urinary tract infections (UTIs) might have differing pathogens or rates of Escherichia coli antibiotic sensitivity and if our physicians managed them in a manner similar or dissimilar to the care provided to younger patients with no complications.

Methods: This was a secondary analysis from patients excluded from a previous retrospective study regarding uncomplicated UTIs.

Results: Twenty-six percent of total patients with UTIs were older than 65 and otherwise medically uncomplicated whereas $21 \%$ were older patients who did have complicating factors. $E$. coli was a pathogen in $81 \%$ of uncomplicated elders' and $54 \%$ of complicated elders' cultures. $E$. coli sensitivity rate to sulfamethoxazole-trimethoprim (SMX/TMP) in both groups was $86 \%$. Physicians were significantly less likely to prescribe SMX/TMP for complicated older patients with complications than for young patients with an uncomplicated UTI $(P=.017)$; there was a significant trend of physicians to be less likely to prescribe SMX/TMP with advancing age in a patient and complications across all 3 groups $(P=.011)$. Antibiotics rarely needed to be changed after cultures.

Conclusions: The presence of $E$. coli on culture in patients with a UTI changes based on medical complications, not age. Being medically complex did not result in reduced sensitivity of $E$. coli to SMX/ TMP but was associated with increased rates of the presence of other pathogens. In our setting, treatment employed with SMX/TMP and without the use of culture and sensitivity may be effective for appropriately selected older women. Prospective studies are needed to determine the optimal approach to management. (J Am Board Fam Med 2009;22:266-71.)

Urinary tract infection (UTI) is a common medical problem for women through out their lifetimes. ${ }^{1}$ Approximately $10 \%$ of postmenopausal women report having had a UTI during the past year. ${ }^{2}$ Evidence-based clinical guidelines have been developed for management of the uncomplicated UTI patient, ${ }^{3,4}$ but substantially less consensus is present for the correct management of older women. Care for women who are over the age of 65 has been

This article was externally peer reviewed.

Submitted 9 June 2008; revised 21 October 2008; accepted 30 October 2008.

From the Department of Family Medicine, Mayo Clinic College of Medicine (MLG, JDB, FDE), Scottsdale, AZ; the Osher Institute, Harvard Medical School (AKK), Boston, MA; and the Division of Biostatistics, Mayo Clinic College of Medicine (ALW), Rochester, MN.

Funding: none.

Previous presentation: This paper was previously presented as a poster at the Society of Teachers of Family Medicine Spring Conference, Chicago, IL, April 26, 2007.

Conflict of interest: none declared.

Corresponding author: Michael L. Grover, DO, Department of Family Medicine, Mayo Clinic College of Medicine, 13737 North 92nd Street, Scottsdale, AZ 85260 (E-mail: grover.michael@mayo.edu). excluded from protocols because age is characterized as a complicating factor. Many older women, however, are not otherwise medically complicated and may have no other anatomic or functional risks.

We previously have reported findings from a retrospective chart review of our management of patients aged 18 to 65 years with uncomplicated UTIs. ${ }^{5}$ At our community-based, university-affiliated residency site, only $30 \%$ of patients had an uncomplicated UTI (ie, they were young and had no complicating features) and were considered eligible for treatment using evidence-based practice guidelines. These guidelines place emphasis on empiric treatment with sulfamethoxazole-trimethoprim (SMX/ TMP) without urine culture and sensitivity testing (UC\&S). Despite this, our physicians ordered urine cultures frequently (76\%). SMX/TMP was used as initial therapy in only $38 \%$ of these low-risk patients; Escherichia coli was sensitive to SMX-TMP in 94\% of culture specimens. Treatment was not changed in any of these young patients with an uncomplicated UTI because of the results of UC\&S. We found that 
antibiotic sensitivity patterns against $E$. coli for these low-risk outpatients were significantly better than from those for inpatients at our hospital, making their potential management within clinical guidelines appropriate. However, of these young patients with uncomplicated UTI, fewer than $25 \%$ received empirical antibiotic treatment without UC\&S as suggested.

It is unclear if age alone is truly a marker of increased risk for UTI complications. The Infectious Disease Society of America's (IDSA) clinical guideline suggests that "studies would be helpful that focus on specific subsegments [of UTI patients], such as the elderly." ${ }^{3}$ We were interested to know if our older female patients might have differing UTI pathogens or increased rates of E. coli antibiotic resistance and if our physicians managed them in a manner similar or dissimilar to the care provided to the younger, uncomplicated patients from our previous report. ${ }^{5}$

The objectives of this study were to evaluate the care of women who were age 65 or older who presented to our office with UTI symptoms and to determine (1) the antibiotic treatments provided and their durations; (2) the frequency of $E$. coli versus other bacteria found on UC\&S testing; and (3) the frequency with which it was necessary to change antibiotic treatments based on UC\&S results.

\section{Methods}

\section{Patients}

This project represents a secondary analysis of data collected about uncomplicated and complicated older patients who had been excluded from participation in our original quality-improvement study based at our family medicine residency program. ${ }^{5}$ Patients were identified by International Classification of Diseases, Ninth Revision, Code 599.0 (UTI). For this analysis, we only included women older than 65 at the time of their first visit for UTI in 2005 .

Two groups were created for comparison: "uncomplicated elders" were women age 65 years or older with no other markers of UTI complication; "complicated elders" included women older than 65 who also had one or more of the following complicating factors as defined in the Institute for Clinical Systems Improvement (ICSI) UTI clinical guideline $^{4}$ : symptoms $>7$ days, documented or reported fever $\left(101.1^{\circ} \mathrm{F}\right)$, nausea or vomiting, con- comitant symptoms or diagnosis of vaginitis, reported or reproduced flank pain, a history of 4 UTIs in the past 12 months, or failure of SMX/ TMP treatment for a UTI in the preceding 4 weeks. Complicated patients also included nursing home patients and patients with functional or anatomic abnormality (polycystic renal disease, nephrolithiasis, neurogenic bladder, diabetes mellitus, immunosuppression, indwelling Foley catheter, or recent urinary tract instrumentation). ${ }^{4}$

\section{Data Collection}

For this analysis, we specifically reviewed pathogens and antibiotic sensitivities of E. coli to SMX/ TMP for each group's UC\&S. We determined rates of antibiotic use, duration of treatments, and the frequency with which we needed to change therapy based on UC\&S results.

\section{Data Analysis}

We developed a data collection sheet for our retrospective chart review on which to record information. Data from these sheets were entered into an Excel database (Microsoft Corp., Redmond, WA) and analyzed using the SAS software package (version 8.2, SAS Institute, Cary, NC). In this secondary analysis, we compared both elder patient groups' data to findings from the uncomplicated UTI group. ${ }^{5}$ Trends in rates across the 3 groups (ordered by advancing age then complications) were evaluated using the Cochran-Armitage trend test. Otherwise, 2-group comparisons were evaluated based on the $\chi^{2}$ test. All calculated $P$ values were 2 -sided and $P<.05$ was considered statistically significant. This project was approved by the Mayo Clinic Foundation Institutional Review Board.

\section{Results}

Two hundred twenty-eight patients seen in the office by physicians for evaluation of symptomatic complaints of a UTI were reviewed. One hundred six $(47 \%)$ of the patients were over the age of 65 . Fifty-nine $(26 \%)$ of the patients were uncomplicated elders and 47 (21\%) were complicated elder patients (see Figure 1). Demographic information regarding the age ranges for patients in each subject group can be seen in Table 1. Although complicated elder patients were slightly older then their 


\section{Uncomplicated UTI patients $(<65 \mathrm{y} / \mathrm{o})$ $30 \%$ of all patients}

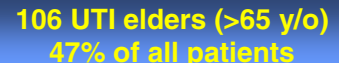

$47 \%$ of all patients

\section{4 complicated young \\ UTI patients \\ $24 \%$ of all patients}

\section{Uncomplicated \\ elders \\ $56 \%$ of elder pts.}

\section{7 complicated \\ elders \\ $44 \%$ of elder pts.}

Figure 1. Flow chart demonstrating creation of patient groups. UTI, urinary tract infection

uncomplicated peers, this difference was not statistically significant $(P=.12)$.

\section{Urine Culture Testing}

Forty-four of 59 (75\%) uncomplicated elders and 37 of 47 (79\%) complicated elder patients had $\mathrm{UC} \& \mathrm{~S}$ testing ordered by their physician; this rate was similar to that seen among uncomplicated UTI patients (52 of $68 ; 76 \%$ ) (Table 2). Rates of cultures showing $E$. coli as a pathogen decreased with progressive age and medical complication $(P<.001)$. Infections in complicated elder patients were significantly more likely to be caused by pathogens other than E. coli (46\%) compared with pathogens found in young uncomplicated patients $(8 \%)$ and uncomplicated elder patients (19\%). The difference in rate of $E$. coli infection between uncomplicated elders and uncomplicated young female patients was not statistically significant.

\section{Sensitivity of E. coli to SMX/TMP}

E. coli sensitivity to SMX/TMP did not change because of advancing age and/or increased medical complications. Both elder groups had E.coli on culture that was sensitive to SMX/TMP $86 \%$ of the time. This is not statistically different from the 94\% sensitivity found in our uncomplicated UTI patients reported previously. ${ }^{5}$

\section{Antibiotic Treatment Used}

Fifty-eight of the 59 uncomplicated elders were treated, along with 42 of the 47 complicated elder patients. Mean treatment durations were 5.7 and 6.4 days for uncomplicated and complicated elder patients, respectively, with a range of 1 day of treatment (for a patient treated with ceftriaxone intramuscularly) to 14 days' duration. Ciprofloxacin was the most-used antibiotic for older patients with UTI; 29 (50\%) uncomplicated elder patients and 20 (48\%) complicated elder patients were treated with it.

We previously reported that in young, uncomplicated patients we used SMX/TMP in 26 of the 68 patients $(38 \%) .^{5}$ In uncomplicated elder patients, SMX/TMP was used in 14 (24\%) compared with $7(17 \%)$ of complicated elder patients. There was a significant trend among physicians to be less likely to prescribe TMP/SMX to patients with advancing age and complications across all 3 groups $(P=.011)$. In particular, physicians were signifi-

Table 1. Average Age and Range for Urinary Tract Infection Patient Groups

\begin{tabular}{lclll}
\hline & & & Age \\
\cline { 3 - 5 } Group & Patients (n) & Mean (SD) & Median & Range \\
\hline Uncomplicated young & 68 & $41.2(14.7)$ & 44.5 & $18-65$ \\
Uncomplicated elder & 59 & $74.8(6.4)$ & 73 & $66-95$ \\
Complicated elder & 47 & $76.7(6.5)$ & 77 & $66-95^{*}$ \\
All patients & 228 & $57.5(21.0)$ & 62.5 & $18-95$ \\
\hline
\end{tabular}

*Complicated elder patients with urinary tract infections were not significantly older than uncomplicated elders $(P=.12)$. 


\begin{tabular}{|c|c|c|c|c|c|c|}
\hline \multirow[b]{2}{*}{ Characteristic } & \multicolumn{2}{|c|}{$\begin{array}{l}\text { Uncomplicated UTI } \\
\qquad(\mathrm{n}=68)\end{array}$} & \multicolumn{2}{|c|}{$\begin{array}{l}\text { Uncomplicated } \\
\text { Elders }(\mathrm{n}=59)\end{array}$} & \multicolumn{2}{|c|}{$\begin{array}{l}\text { Complicated Elders } \\
(\mathrm{n}=47)\end{array}$} \\
\hline & $\mathrm{N}(\%)$ & $95 \% \mathrm{CI}$ & $\mathrm{N}(\%)$ & $95 \% \mathrm{CI}$ & $\mathrm{N}(\%)$ & $95 \% \mathrm{CI}$ \\
\hline UC\&S testing ordered & $52(76)$ & $66-87^{*}$ & $44(75)$ & $83-86^{*}$ & $37(79)$ & $67-90^{*}$ \\
\hline Mixed flora/contamination present in urine culture & \multicolumn{2}{|c|}{$\mathrm{n}=52$} & \multicolumn{2}{|c|}{$\mathrm{n}=44$} & \multicolumn{2}{|c|}{$\mathrm{n}=37$} \\
\hline Yes & \multicolumn{2}{|l|}{$11(21)$} & \multicolumn{2}{|l|}{$12(27)$} & \multicolumn{2}{|l|}{$9(24)$} \\
\hline No & \multicolumn{2}{|l|}{$38(73)$} & \multicolumn{2}{|l|}{$27(61)$} & \multicolumn{2}{|l|}{$26(70)$} \\
\hline No growth & \multicolumn{2}{|l|}{$3(6)$} & \multicolumn{2}{|l|}{$5(11)$} & \multicolumn{2}{|l|}{$2(5)$} \\
\hline Type of pathogens grown & \multicolumn{2}{|c|}{$\mathrm{n}=38$} & \multicolumn{2}{|c|}{$\mathrm{n}=27$} & \multicolumn{2}{|c|}{$\mathrm{n}=26$} \\
\hline E. coli & $35(92)$ & $79-98$ & $22(81)$ & $62-94$ & $14(54)$ & $33-73$ \\
\hline E. coli only & 32 & & 19 & & 14 & \\
\hline E. coli and other bacteria & 3 & & 3 & & 0 & \\
\hline Other bacteria only & $3(8)$ & $2-21$ & $5(19)$ & $6-38$ & $12(46)$ & $27-67$ \\
\hline E. coli sensitivity to TMP/SMX & $\begin{array}{l}33(94) \\
(\mathrm{n}=35)\end{array}$ & $81-99^{*}$ & $\begin{array}{l}19(86) \\
(\mathrm{n}=22)\end{array}$ & $65-97^{*}$ & $\begin{array}{l}12(86) \\
(\mathrm{n}=14)\end{array}$ & $57-98^{*}$ \\
\hline
\end{tabular}

*95\% CI show the proportion of patients who had urine culture and sensitivity testing ordered that were calculated using normal theory methods; all other $95 \%$ CIs were calculated using an exact method of obtaining a confidence interval for a binomial parameter. UTI, urinary tract infection; UC\&S, of urine culture and sensitivity; TMP/SMX, trimethoprim/sulfamethoxazole.

cantly less likely to prescribe SMX/TMP for complicated elder patients than for young patients with uncomplicated UTIs $(P=.017)$. Antibiotic treatment was changed because of bacterial resistance found on UC\&S reports in 3 of the 58 uncomplicated elders (5\%) and 1 of the 42 complicated elders $(2 \%)$ that were treated.

\section{Discussion}

The ICSI clinical guideline recommends classifying patients $>65$ years old as being complicated and that a provider evaluation is necessary. ${ }^{4} \mathrm{We}$ were interested in evaluating whether the infections in otherwise uncomplicated older women were similar to their younger peers and whether it seemed reasonable, therefore, to treat them empirically, without UC\&S, if they had similar pathogens and sensitivity patterns.

We found that in our uncomplicated elder group (ie, without complicating factors other than age) the frequency of UTIs being caused by E. coli was similar to that of younger uncomplicated patients, as was its sensitivity to SMX/TMP. Because of the relatively small number of patients with $E$. coli in this study there was limited statistical power to detect a difference in the sensitivity rates between the groups, as evidenced by the wide $95 \%$ confidence intervals. E. coli did, however, become less likely to be the cause of UTIs in our complicated elder patients, perhaps making obtaining a
UC\&S more critical. Although it would seem that it might be of clinical utility to identify the other organisms that caused UTI in these medically complicated seniors, very few needed to have their treatments changed because of antibiotic resistance.

Our physicians did provide antibiotic treatments for progressively longer durations based on patient age and complication as has been traditionally recommended. The Infectious Disease Society of America's review of the literature suggested that older women tended to have lower bacteriuria eradication rates and supported the use of a 7-day antibiotic regimen for older women. ${ }^{3}$ The ICSI guidelines state that "there is little literature documentation of efficacy of short-course therapy" in this age group. ${ }^{4} \mathrm{~A}$ recently updated Cochrane review now recommends that short-term (3- to 6-day) treatment of uncomplicated UTIs in older women is as effective as long-term treatment (7 to 14 days). ${ }^{6}$ Comparing the efficacy of short versus longer duration antibiotic treatment was not an objective of this study and therefore we can make no specific recommendations on this subject.

Our physicians most frequently prescribed ciprofloxacin for treatment of UTIs in elderly patients. This seems to be supported by a study that compared a 10-day course of ciprofloxacin with a 10-day course of SMX/TMP. ${ }^{7}$ Gomolin et $\mathrm{al}^{7}$ found a $96 \%$ bacteriologic eradication rate with 
ciprofloxacin compared with an $80 \%$ eradication rate with SMX/TMP for their 3 most common isolates. Although this seems impressive, the patient population Gomolin et $\mathrm{al}^{7}$ studied was quite diverse. More than half of the patients enrolled were in nursing facilities. In our study, we enrolled only ambulatory patients who were able to be seen in the office. Our patient group of interest was unique and specific (patient without risks of complication other then age) whereas they looked at a much larger cross-section of elderly patients.

\section{Limitations}

This study provides some initial, although admittedly preliminary, evidence that suggests that women older than 65 with otherwise uncomplicated UTIs might be able to be treated the same as younger women (using SMX/TMP without the use of a culture). Our findings certainly do support previous recommendations that women with complicated UTIs need to be managed with in-person evaluation and that the appropriateness of initial antibiotic treatment choices should be confirmed by obtaining a urine culture. The retrospective nature of this study demonstrates the need to perform future prospective studies on this topic. We can conclude that among evaluated patients with diagnosed UTI (albeit with a single-billed ICD-9 code of 599.0) and no noted complicating factors other than E. coli sensitive to SMX/TMP predominated. Our study data start with a given clinical diagnosis made with an office visit in retrospect. Patients called or presented to their physicians with symptoms which may be less likely to, in fact, represent simple cystitis in older women.

Our findings remind us that clinical considerations matter more than the absolute age of the patient. Although our 2 groups of elder patients did not differ statistically in their mean age or age range, it might be expected that with increasing age there could be fewer and fewer patients without functional impairments or comorbidities. The primary care physician's knowledge of each patient's individual risks for UTI complication, aside from her chronologic age, will play a major role in the decision to attempt empiric treatment without culture.

Comparison with other studies is difficult because we did not measure eradication rates directly but simply reviewed whether resistant bacteria were found. Only a few older women needed to have treatment changed because of their culture results. We did not, however, review whether any of these women had temporary improvement with a rapid return of symptoms or a failure of treatment altogether (with persistence or escalation in their symptoms, for example, to pyleonephritis). A clinical cure with eradication of bacteriuria may not have occurred in some of our patients despite the pathogen on UC\&S being sensitive to the prescribed antibiotic.

In addition, there may be other clinical factors that contributed to a physician's decision about antibiotic choice for which we cannot account. We did not determine a patient's medication allergy history or whether renal insufficiency or other clinical factors might have influenced treatment. Future prospective studies would be better suited to determine the clinical reasoning for those decisions.

We were surprised to discover that older patients with complications did not have differences in the rate at which E. coli was sensitive to SMX /TMP. Based on these findings, we suggest that our departmental physicians can reconsider SMX/ TMP in appropriately selected older patients who are otherwise not medically complicated as a firstline antimicrobial agent. The IDSA recommends its use in areas where the prevalence of resistance to the drug among E. coli strains causing cystitis is $<20 \%$. ${ }^{3}$ Rates were $<20 \%$ for all 3 of the patient groups included in this study. We suggest that other physicians determine the bacterial prevalence and antibiotic sensitivity patterns in their own settings to determine whether this approach is appropriate for their own patient population.

\section{Conclusions}

We found that elder women who were not otherwise complicated by medical or functional risks had infections that were frequently caused by $E$. coli and were favorably sensitive to SMX/TMP (in manner statistically similar to younger, uncomplicated patients with UTIs). These findings provide some initial preliminary evidence that, for our ambulatory outpatient practice, treatment employed with SMX/TMP and without the use of UC\&S may be effective in the treatment of uncomplicated UTIs in elder women because UTI pathogens seem to change primarily because of medical complication, not age. Future prospective studies are needed to 
further explore the boundaries of existing clinical guidelines about who might be safely and effectively treated without a culture or with simpler, less expensive antibiotic therapy.

We wish to acknowledge the contributions of Michael Bryan, DO; Sean Donahue, DO; and Anne-Marie Warner, MD, for their efforts in data collection for this project.

\section{References}

1. Jackson SL, Boyko EJ, Scholes D, Abraham L, Gupta K, Fihn SD. Predictors of urinary tract infection after menopause: a prospective study. Am J Med 2004;117:903-11.

2. Foxman B, Barlow R, D'Arcy H, Gillespie B, Sobel JD. Urinary tract infection: self-reported incidence and associated costs. Ann Epidemiol 2000;10:50915.

3. Warren JW, Abrutyn E, Hebel JR, Johnson JR, Schaeffer AJ, Stamm WE. Guidelines for antimicrobial treatment of uncomplicated acute bacterial cystitis and acute pyelonephritis in women. Infectious
Diseases Society of America (IDSA). Clin Infect Dis 1999;29:745-58.

4. Kasten M, Olson D, Marroquin R, et al. Institute for Clinical Systems Improvement Health Care Guideline: Uncomplicated urinary tract infection in women. Available from: http://www.icsi.org/urinary_ tract_infection/uncomplicated_urinary_tract_infection_ in_women_2.html. Accessed 21 January 2009.

5. Grover ML, Bracamonte JD, Kanodia AK, Bryan MJ, Donahue SP, Warner AM, Edwards FD, Weaver AL. Assessing Adherence to Evidence Based Guidelines for the Diagnosis and Management of Uncomplicated Urinary Tract Infections. Mayo Clin Proc 2007;82:181-5.

6. Lutters M, Vogt-Ferrier NB. Antibiotic duration for treating uncomplicated, symptomatic lower urinary tract infections in elderly women. Cochrane Database Syst Rev 2008;(3):CD001535.

7. Gomolin IH, Siami PF, Reuning-Scherer J, Haverstock DC, Heyd A, the Oral Suspension Study Group. Efficacy and safety of ciprofloxacin oral suspension versus trimethoprim-sulfamethoxazole oral suspension for treatment of older women with acute urinary tract infection. J Am Geriatr Soc 2001;49: $1606-13$. 